\title{
Traumatic Sinus of Valsalva Aneurysm Repaired by Partial David Operation
}

\author{
Hiroshi Seki ${ }^{1} \quad$ Michael Borger $^{1}$ Friedrich Mohr ${ }^{1}$ Martin Misfeld ${ }^{1}$ \\ ${ }^{1}$ Heart Center Leipzig, Leipzig, Germany \\ Address for correspondence Hiroshi Seki, MD, Heart Center Leipzig, \\ Struempellstr.39, Leipzig 04289, Germany \\ Thorac Cardiovasc Surg Rep 2014;3:13-15. \\ (e-mail: shunryou12111329111@yahoo.com).
}

\begin{abstract}
Keywords

- aortic disease

- cardiovascular surgery

- trauma

We report a case of chronic type A dissection and aneurysm of the sinus of Valsalva after a motor bike accident which remained undetected for 20 years. It was successfully treated with a modified valve-preserving technique. The noncoronary sinus formed a $4.5 \times 5.0 \mathrm{~cm}$ large and heavily calcified aneurysm, compressing the right atrium and right coronary artery. The pathology was limited to the noncoronary sinus area and here was the annulus dilatated but the remaining sinuses and annulus and the cusps were intact. We have therefore performed a one-third or partial-David procedure with a satisfactory result.
\end{abstract}

\section{Introduction}

Traumatic aortic injury is a rare, often acute lethal condition varying widely in extent. It commonly occurs in the area of the aortic isthmus or the aortic root due to anatomical reasons. If undetected, small lesions which did not present in the acute phase may also develop into chronic aneurysm. We report here a case of a chronic type A dissection which was assumed to have occurred after a traffic accident and which was successfully treated with a modified valve-preserving technique.

\section{Case Report}

A 44-year-old man with a history of chest trauma was referred to our hospital after an aneurysm of the sinus of Valsalva was detected by chance in a computed tomography (CT) scan. He had been severely injured in a motorcycle accident in 1990, suffering multiple fractures. However, he had no complaints at the time of reference. There was no positive family history of aortic dissections or connective tissue disorders. Echocardiography revealed an enlargement of the root in the noncoronary sinus up to $55 \mathrm{~mm}$ in size including severe calcification of the whole noncoronary sinus and mild aortic valve regurgitation. The $\mathrm{CT}$ scan showed a 2- to 3-mm-thick calcification layer inside the noncoronary sinus extending into the right coronary ostium ( - Fig. 1). Moreover, there was a 12-mm large evagination near the annulus which was also surrounded by calcification, suggesting a covered rupture at the time of the initial trauma. A preoperative coronary angiography showed 50\% stenosis of the left anterior descending artery and a total occlusion of the right coronary artery at the level of the ostium. The right coronary system was collateralized from the left coronary artery system. The patient was operated electively through a median sternotomy. Cardiopulmonary bypass (CPB) was established via ascending aortic perfusion and right atrial drainage. Blood cardioplegia was administered initially via the aortic root followed by intermittent direct selective cardioplegia into the left coronary ostium. First of all, a bypass operation from the left internal mammary artery to the left anterior descending artery was performed. Direct inspection revealed the aneurysm of the noncoronary sinus to be extremely dilated $(5 \times 4.5 \mathrm{~cm})$, heavily calcified and stiff, resulting in the anticlockwise rotation of the noncoronary sinus toward the left. Calcification extended into the ostium of the right coronary artery, which was positioned very close to the commissure between the right and noncoronary sinus. After the removal of the calcification bowl (-Fig. 2), the remaining right atrial roof was reinforced with a bovine pericardial patch because the aneurysm had led to extreme thinning of the atrial wall. A partial David's operation was performed by tailoring a 26-mm Gelweave prosthesis (Inchinnan, Scotland, received

November 9, 2013 accepted after revision April 22, 2014

published online

July 16, 2014
Dol http://dx.doi.org/ 10.1055/s-0034-1383431. ISSN 2194-7635. (c) 2014 Georg Thieme Verlag KG
Stuttgart . New York

License terms

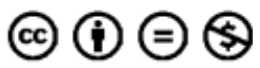




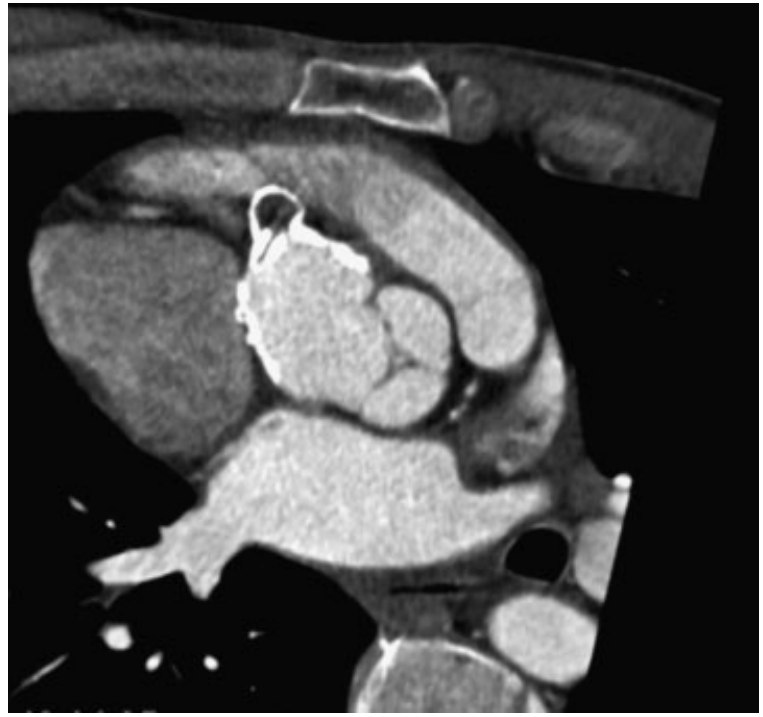

Fig. 1 Computed tomography scan showing a 2- to 3-mm-thick calcification layer inside the noncoronary sinus extending near the right coronary ostium. In addition, a 12-mm evagination near the annulus was also surrounded by calcification, suggesting a covered rupture at the time of trauma.

UK) and producing a 4-cm-wide tongue, which was used for the reconstruction of the noncoronary sinus and which covered the calcified right coronary ostium as well and obliged an implantation of the extended right coronary artery into the prosthesis. The prosthesis was fixed subannularly with a felt-pledged Tevdek suture (Illinois, United States), shortening the dilated annulus in this part. The Dacron tube (Inchinnan, Scotland, UK) stabilized the noncoronary annulus from the area of the left to nonsubcommissural trigone to the right to nonsubcommissural trigone (-Fig. 3). The commissures were positioned at an appropriate position in the partial graft resulting in a one-third or partial David's procedure. The right coronary artery, which was totally occluded and squeezed due to the aneurysm in its proximal part and therefore no longer feasible to reimplant was extended by $4 \mathrm{~cm}$ with a short saphenous vein graft as an interponate. Following additional supracommissural replacement of the ascending aorta without circulatory arrest and reimplantation of the extended right coronary artery, the patient was weaned from the CPB and transferred to the intensive care

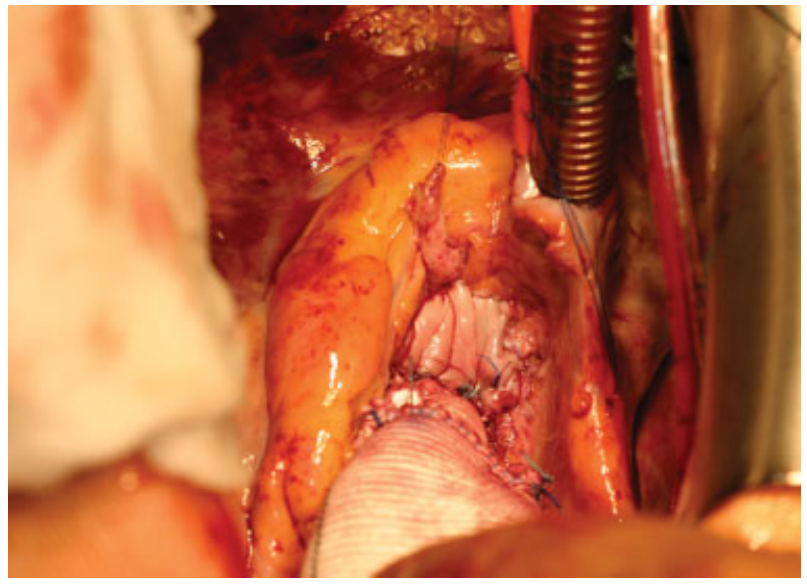

Fig. 3 The right atrial roof was reinforced with a bovine pericardial patch. The tailored Dacron prosthesis stabilized the noncoronary annulus from the area of the left to nonsubcommissural trigone to the right to nonsubcommissural trigone.

unit (ICU), where he was extubated after a further uneventful course 4 hours postoperatively. The CPB time was 137 minutes and cross-clamp time was 122 minutes. The ICU stay was 6 hours. Postoperative echocardiography performed on the 5 th postoperative day showed an excellent result after the partial David's operation with no residual aortic valve insufficiency and good systolic function. The patient was transferred to the rehabilitation center on the 8th postoperative day.

\section{Comments}

A traumatic dissection is a rare condition which could cause life-threatening acute critical situations such as ischemia, pericardial tamponade, or rupture. If it is undetected, it could lead to chronic aneurysm with the risk of rupture.

In our case, the aneurysm of the noncoronary sinus which most probably originated from a covered rupture and dissection in the aortic root led to annular dilatation and deformity of the aortic root, causing aortic valve regurgitation. This condition could have been treated in other surgical ways, one option being a Bentall and De Bono ${ }^{1}$ procedure. However, our patient had a strong wish to avoid anticoagulation therapy, and as there were no pathological changes to the cusps

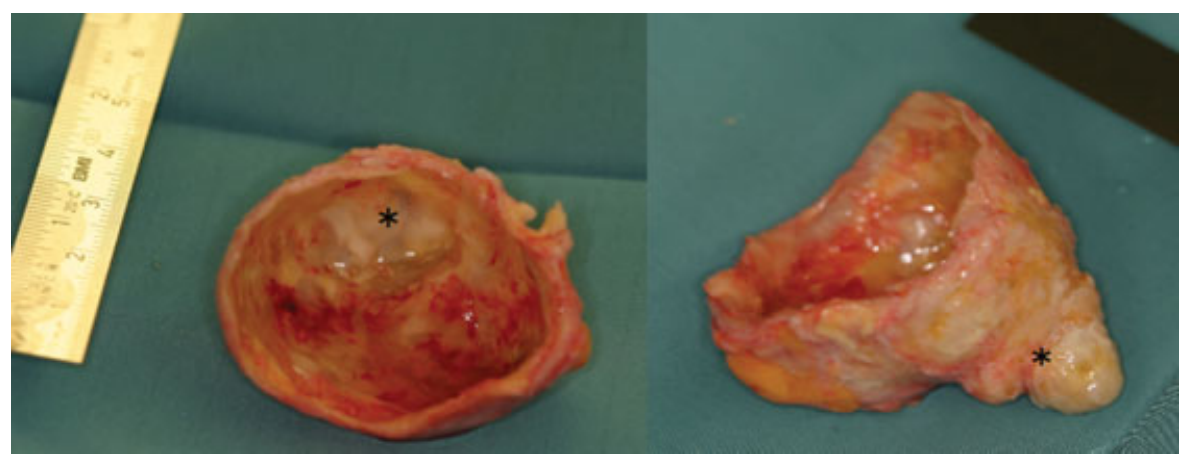

Fig. 2 A large calcification shell $(5 \times 4.5 \mathrm{~cm})$ including a small evagination (marked *) was removed from the noncoronary sinus of Valsalva. 
themselves, we decided to perform this valve-sparing procedure.

There are basically two types of aortic valve-sparing operations: the remodeling technique initially described by Yacoub et $\mathrm{al}^{2}$ and the reimplantation technique introduced by David et al. ${ }^{3}$ Various modifications of these operations such as partial valve-sparing procedures have been developed as well. Realization that dilatation of the root is frequently limited to the noncoronary sinus and the wish to avoid unnecessary mobilization of the coronary arteries prompted us and other authors to preserve the intact sinuses and to replace the pathological sinus selectively. ${ }^{4-6}$ Charitos et al reported the feasibility and safety of the partial root-preserving procedure in the remodeling technique. ${ }^{6}$ However, they also observed significant dilatation at sinus and ST junction level in their patients following the use of the partial remodeling technique instead of the complete remodeling of all three sinuses, which may be related to increased stress in the remaining native structures because of the presence of an incompatible material. They, therefore, emphasized that it is essential to fully understand the pathology of the root dilatation (including by inspecting the ST junction level) before applying this technique. In our case, we observed limited aneurysmal formation in the noncoronary sinus and also limited dilatation of the annulus in this area. We, therefore, applied a valve-preserving procedure as a partial reimplantation technique to avoid further widening. Modifying a standard aortic valve-preserving technique to repair the limited anatomical distortion of the aortic root was feasible in our case of posttraumatic, noncoronary sinus aneurysm formation, and aortic regurgitation. Because the aortic valve function was preserved, lifelong anticoagulation treatment was avoided.

Conflict of Interest

There is no conflict of interest to be disclosed.

\section{References}

1 Bentall H, De Bono A. A technique for complete replacement of the ascending aorta. Thorax 1968;23(4):338-339

2 Yacoub MH, Gehle P, Chandrasekaran V, Birks EJ, Child A, RadleySmith R. Late results of a valve-preserving operation in patients with aneurysms of the ascending aorta and root. J Thorac Cardiovasc Surg 1998;115(5):1080-1090

3 David TE, Ivanov J, Armstrong S, Feindel CM, Webb GD. Aortic valve-sparing operations in patients with aneurysms of the aortic root or ascending aorta. Ann Thorac Surg 2002;74(5): S1758-S1761, discussion S1792-S1799

4 Cerillo AG, Farneti PA, Mariani M, Gasbarri T, Solinas M, Glauber M. Valve-sparing replacement of the noncoronary sinus of Valsalva in patients with a bicuspid aortic valve. Ann Thorac Surg 2007;84(5): 1774-1776

5 Gerosa G, Pontarollo S, Iliceto S, di Marco F. An alternative technique for aortic root remodeling in patients with bicuspid aortic valve. J Thorac Cardiovasc Surg 2007;133(1):249-250

6 Charitos EI, Stierle U, Sievers HH, Misfeld M. Valve-sparing aortic root remodeling with partial preservation of the intact native aortic sinuses. Eur J Cardiothorac Surg 2009;36(3):589-591 